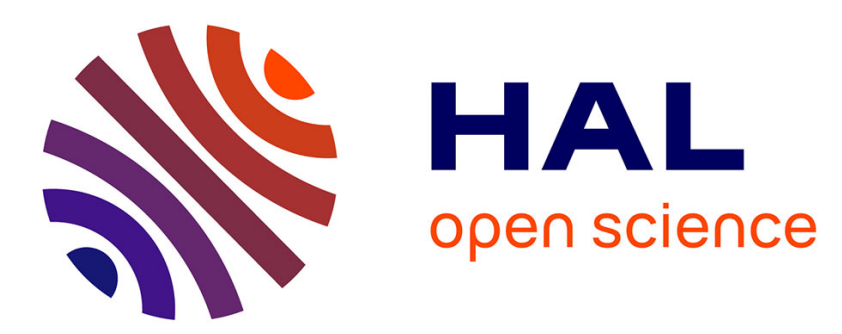

\title{
Multiplicity-Induced-Dominancy extended to neutral delay equations: Towards a systematic PID tuning based on Rightmost root assignment
}

Dan Ma, Islam Boussaada, Catherine Bonnet, Silviu-Iulian Niculescu, Jie Chen

\section{To cite this version:}

Dan Ma, Islam Boussaada, Catherine Bonnet, Silviu-Iulian Niculescu, Jie Chen. Multiplicity-InducedDominancy extended to neutral delay equations: Towards a systematic PID tuning based on Rightmost root assignment. ACC 2020 - American Control Conference, Jul 2020, Denver, United States. 10.23919/acc45564.2020.9147341 . hal-02479679

\section{HAL Id: hal-02479679 \\ https://hal.science/hal-02479679}

Submitted on 14 Feb 2020

HAL is a multi-disciplinary open access archive for the deposit and dissemination of scientific research documents, whether they are published or not. The documents may come from teaching and research institutions in France or abroad, or from public or private research centers.
L'archive ouverte pluridisciplinaire HAL, est destinée au dépôt et à la diffusion de documents scientifiques de niveau recherche, publiés ou non, émanant des établissements d'enseignement et de recherche français ou étrangers, des laboratoires publics ou privés. 


\title{
Multiplicity-Induced-Dominancy extended to neutral delay equations: Towards a systematic PID tuning based on Rightmost root assignment
}

\author{
Dan Ma and Islam Boussaada and Catherine Bonnet and Silviu-Iulian Niculescu and Jie Chen
}

\begin{abstract}
Recently, the conditions on a multiple spectral value to be dominant for retarded time-delay system with a single delay were deeply explored. Such a property was called Multiplicity-Induced-Dominancy. It was then exploited in the design of delayed stabilizing controllers. As a matter of fact, the approach is merely a delayed-output-feedback where the candidates' delays and gains result from the manifold defining the maximal multiplicity of a real spectral value. This can also be seen as a pole-placement method, which unlike methods based on finite spectrum assignment does not render the closed loop system finite dimensional but consists in controlling its rightmost spectral value. This work aims at extending such a design approach to time-delay systems of neutral type occurring in the classical problem of PID stabilizing design for delayed plants. More precisely, the controller's gains $\left(k_{i}, k_{p}, k_{d}\right)$ are tuned using the intentional multiplicity's algebraic constraints allowing to the stabilization of unstable delayed plants. The specificity, of such a design is related to the analytical assignment of the closed-loop solution's decay rate.
\end{abstract}

Keywords-Time-delay systems, Neutral systems, Asymptotic stability, $\alpha$-stability, Frequency domain analysis, PID tuning, Pole placement, Rightmost root, Delay margin.

\section{INTRODUCTION}

Linear systems with commensurate delays (all delays are multiple of a nominal delay) are described in the Laplace domain by transfer functions involving quasi-polynomials and then possibly admit an infinite number of poles. Studying the stability properties of retarded systems (they admit a finite number of poles in any right half-plane) is much easier than studying those of neutral systems which may have an infinite number of poles, in chains asymptotic to vertical axes possibly located in the open right half-plane or clustering the imaginary axis from left or right. Both situations prevent to get exponential stability for these systems. A subclass of

Dan Ma is with the College of Information Science and Engineering, State Key Laboratory of Synthetical Automation for Process Industries, Northeastern University, Shenyang, China, madan@mail. neu. cn. edu

Islam Boussaada is with Inria Saclay, DISCO Team \& IPSA \& Laboratoire des Signaux et Systèmes, Université Paris Saclay, CNRS-CentraleSupélec-Université Paris Sud, 3 rue Joliot-Curie, 91192 Gif-sur-Yvette cedex, France islam.boussaadaecentralesupelec.fr

Catherine Bonnet is with Inria Saclay \& Laboratoire des Signaux et Systèmes, Université Paris Saclay, CNRS-CentraleSupélec-Université Paris Sud, 3 rue Joliot-Curie, 91192 Gif-sur-Yvette cedex, France catherine.bonnetecentralesupelec.fr

Silviu-Iulian Niculescu is with Laboratoire des Signaux et Systèmes, Université Paris Saclay, CNRS-CentraleSupélec-Université Paris Sud, 3 rue Joliot-Curie, 91192 Gif-sur-Yvette cedex, France, silviu.niculescudcentralesupelec.fr

Jie Chen is with the Department of Electronic Engineering, City University of Hong Kong, Hong Kong, China, jichen@ cityu. edu.hk

Manuscript received September 26th, 2019 neutral systems of interest is the one with all asymptotic axes in the open left half-plane guaranteing that there is a finite number of poles in an extended right half-plane. For such systems, the concept of $\alpha$-stability) will play an important role.

In the Laplace domain, a number of effective methods have been proposed, see for instance [1], [2], [3], [4], [5], [6], [7], [8].

Even with the significant advances that have been reported on the topic of Delay systems, the question of determining conditions on the equation parameters that guarantee asymptotic stability of solutions of linear time-invariant time-delay systems remains an open question.

Once stability conditions are established; further questions related to performance occur. What is about the estimation of the corresponding rightmost roots of the system characteristic equation? Such a rightmost root corresponds to the so-called $\alpha$-stability problem, itself is related to the solution's decay rate. Also a fundamental measure of robust stabilization against uncertain time delays is the so-called delay margin, which addresses a central issue in the study of feedback stabilization of time-delay systems: What is the largest range of delay so that there exists a single controller that can stabilize the delay plant within that entire range? This question is also longstanding and remains open except in particular cases, see for instance [9].

PID controllers have been extensively used to control and regulate industrial processes which are typically modeled by reduced-order dynamics. In [9], the delay margin achievable using PID controllers for reduced order linear time-invariant (LTI) systems subject to variable, unknown time delays is investigated. An explicit expressions of the exact delay margin is carried out and its upper bounds achievable by a PID controller for low-order delay systems with unknown constant and possibly time-varying delays. The effect of non minimum phase zeros is also investigated and the fundamental limits of delay within which a PID controller may robustly stabilize a delay process is emphasized.

In recent works, the characterization of multiple spectral values for time-delay systems of retarded type were established using a Birkhoff/Vandermonde-based approach; see for instance [10], [11], [12], [13]. In particular, in [11], it is shown that the admissible multiplicity of the zero spectral value is bounded by the generic Polya and Szegö bound denoted $P S_{B}$, which is merely the degree of 
the corresponding quasipolynomial ${ }^{1}$, see for instance [14]. In [10], it is shown that a given crossing imaginary root with a non vanishing frequency never reaches $P S_{B}$ and a sharper bound for its admissible multiplicities is established.

Moreover, in [13], the manifold corresponding to a multiple root for scalar time-delay equations defines a stable manifold for the steady state. An example of a scalar retarded equation with two delays is studied in [10] where it is shown that the multiplicity of real spectral values may reach the $P S_{B}$. In addition, the corresponding system has some further interesting properties: (i) it is asymptotically stable, (ii) its spectral abscissa (rightmost root) corresponds to this maximal allowable multiple root located on the imaginary axis. Such observations enhance the outlook of further exhibiting the existing links between the maximal allowable multiplicity of some negative spectral value reaching the quasipolynomial degree and the stability of the trivial solution of the corresponding dynamical system. This interesting property induced by multiplicity appears also in optimization problems since such a multiple spectral value is indeed the rightmost root, see also [15]. Also notice that the property was already observed in [16], where a tuning strategy is proposed for the design of a delayed Proportional-Integral controller by placing a triple real dominant root for the closed-loop system. However, the dominancy is only checked using a Mikhailov curve and QPmR toolbox, see for instance [17].

It is worth noting that the rightmost root for quasipolynomial function corresponding to stable retarded time-delay systems (also in the neutral case under some assumptions) is actually the exponential decay rate of its time-domain solution, see for instance [18] for an estimate of the decay rate for stable linear delay systems. To the best of our knowledge, the first time an analytical proof of the dominancy of a spectral value for the scalar equation with a single delay was presented in [19]. The dominancy property is further explored and analytically shown in scalar delay equations in [13], then in second-order systems controlled by a delayed proportional is proposed in [20], [21] where its applicability in damping active vibrations for a piezo-actuated beam is proved. An extension to the delayed proportional-derivative controller case is studied in [22] where the dominancy property is parametrically characterized. We emphasize that the idea of using roots assignment for controller-design for time-delay system is not new. As a matter of fact, an analytical/numerical stabilization method for retarded timedelay systems related to the classical pole-placement method for ordinary differential equations is proposed in [23], see also [24] for further insights on pole-placement methods for retarded time-delays systems with proportional-integralderivative controller-design.

A stimulating idea from [25], which consists in exploiting the properties of Vandermonde matrices to control one chain of integrators by delay blocks, enticed the authors in [12] and

\footnotetext{
${ }^{1}$ The quasipolynomial degree is exactly the number of the involved polynomials plus their degree minus one
}

[26] to extend the investigation of such structured matrices. A complete characterization of the root at the origin can be found in [12], [26]. It is shown that the multiplicity of such a root for a generic system is bounded by the degree of the associated quasipolynomial, i.e, the sum of the number of its polynomials plus their respective degrees minus one. This bound was already depicted in [14]. Furthermore, in the sparse case, a sharper bound is established in [12] exhibiting the link between the maximal multiplicity and the number of the non vanishing parameters rather than the degree of the quasipolynomial.

The present work is a natural continuation of [12], [26], [11], it aims at proposing a systematic PID controller tuning to achieve the asymptotic stability of a reduced order linear time-invariant dead-time plant by extending and exploiting the Multiplicity-Induced-Dominancy property for quasipolynomial functions corresponding to neutral systems with single delay.

The remaining paper is organized as follows. Some prerequisites in complex analysis and the problem formulation are presented in section 2 . The main results are presented in Section 3 where a PID tuning based on the MID property is provided. Section 4 illustrate the obtained result. A conclusion ends the paper.

\section{PREREQUisites AND PROBLEM FORMULATION}

The principle of the argument is a consequence of Cauchy theorem and connects the winding number of a curve with the number of zeros and poles of a given complex variable function inside that curve. More precisely, it asserts that the integral of the ratio of the single valued complex variable functions $f^{\prime}(z) / f(z)$ on a single passage along a closed path in the positive sense (in counter clockwise direction) gives exactly $2 i \pi(N-P)$ where $N$ (respectively $P$ ) is the sum of multiplicities of zeros (respectively of poles) of the function $f$ enclosed in such a contour. A natural and direct application of the principle of the argument is the stability analysis of dynamical systems. As a matter of fact, in frequency domain, showing the asymptotic stability of the trivial solution of a given dynamical system amounts to prove that the zeros of the corresponding characteristic equation are located in the open left-half complex plane. The principle of argument is applied to determine the stability of closed loop system by choosing a closed path which encircles the entire right half complex plane in counter clockwise direction.

The next proposition follows directly from [27] and [28, Prop. 2.1] which gives an explicit localisation of the spectrum chain's asymptote for quasipolynomial functions corresponding to the following neutral systems.

$$
G(s)=\frac{r(s)}{Q_{0}(s)+Q_{\tau}(s) \mathrm{e}^{-s \tau}}
$$

such that $\operatorname{deg}(r) \leq \operatorname{deg}\left(Q_{0}\right)=\operatorname{deg}\left(Q_{\tau}\right)$.

Proposition 1. Let $\alpha=\lim _{|s| \rightarrow \infty} Q_{\tau}(s) / Q_{0}(s)$

1) If $|\alpha|<1$ then the poles of $G$ of large modulus are asymptotic to a vertical line $\Re(s) \approx \log (|\alpha|) / \tau$ in the 


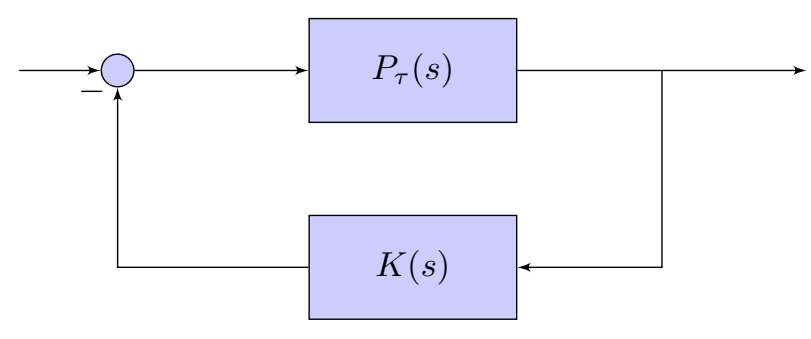

Fig. 1. A feedback control system

left-half plane. The number of poles of $G$ in the right of $\Re(s)=\log (|\alpha|) / \tau+\epsilon$ is finite for any fixed $\epsilon>0$.

2) If $|\alpha|>1$ then $G$ has infinitely many unstable poles, asymptotic to a vertical line $\Re(s) \approx \log (|\alpha|) / \tau$ which is the right-half plane.

So, under the condition $|\alpha|<1$ one is able to prove that at most a finite number of poles of $G$ are located in the righthalf plane. By scaling, this property extends to any parallel to the imaginary axis located at the right of the spectrum chain's asymptote.

Remark. The above result naturally apply to any transfer function

$$
G(s)=\frac{r(s) \mathrm{e}^{-s T}}{Q_{0}(s)+Q_{\tau}(s) \mathrm{e}^{-s \tau}} \quad \text { for any } \quad T>0 .
$$

\section{A. Problem formulation}

Consider the feedback control system depicted in Figure.1 where $P(s)$ is a reduced order plant which is subject to an unknown delay. Thus, the corresponding transfer function is given by

$$
P_{\tau}(s)=P_{0}(s) e^{-\tau s}
$$

where $P_{0}(s)$ is a delay-free plant. Our aim in this paper is to provide an easy way in tuning the standard PID gains $\left(k_{i}, k_{p}, k_{d}\right)$ achieving the stabilization of the closed-loop system. More precisely, the approach consists in intentionally impelling an appropriate multiplicity for a given root of the quasipolynomial function such that the induced algebraic constraints on the quasipolynomial's coefficients leads to robustly stabilize the considered unstable delayed plant. This approach showed its efficiency in studying stability and stabilization of retarded time-delay systems. However, in the present paper context, the obtained characteristic equation in closed-loop system corresponds to a delay system of neutral type, a class for which the stability analysis becomes more involved. As a matter of fact, the structure of the controller we consider is given by:

$$
K_{P I D}(s)=k_{p}+k_{d} s+\frac{k_{i}}{s},
$$

\section{MAIN RESULTS}

\section{A. Robust PID stabilizing design for first-order delayed plants}

First-order plants are typical benchmarks usually considered for controlling industrial processes. In this aim, let consider $P_{0}(s)=\frac{1}{s-p}$ where $p$ is a positive (instable) pole.
It is shown in [29] that the delay margin $\tau_{P I D}=\frac{2}{p}$, see also [9]. The resulting closed-loop plant is given by:

$$
M(s)=\frac{\left(k_{p} s+k_{i}+s^{2} k_{d}\right) \mathrm{e}^{-s \tau}}{s^{2}-s p+\mathrm{e}^{-s \tau} k_{p} s+\mathrm{e}^{-s \tau} k_{i}+\mathrm{e}^{-s \tau} s^{2} k_{d}} .
$$

Since we are dealing with stability aim, le us focus on the corresponding characteristic equation:

$$
\left\{\begin{aligned}
\Delta(s) & =Q_{0}(s)+Q_{\tau}(s) \mathrm{e}^{-s \tau} \quad \text { where } \\
Q_{0}(s) & =s^{2}-s p \quad \text { and } \quad Q_{\tau}(s)=k_{d} s^{2}+k_{p} s+k_{i} .
\end{aligned}\right.
$$

The following theorem provides a bound on the multiplicity of the corresponding zeros as well as a systematic manier to tune the parameters $k_{p}, k_{i}, k_{d}$ such that the closed-loop system becomes stable.

Theorem III.1. i) For arbitrary real parameters $k_{p}, k_{i}, k_{d}$ and arbitrary positive delay $\tau$, the multiplicity of a given root of the quasipolynomial function (6) is bounded by 4.

ii) The quasipolynomial (6) admits a multiple real spectral value at

$$
s_{ \pm}=\frac{-\tau p-6 \pm \sqrt{\tau^{2} p^{2}+12}}{2 \tau}
$$

with algebraic multiplicity 4 if, and only if,

$$
\left\{\begin{array}{l}
k_{d}=\frac{\left(4+2 \tau s_{ \pm}-\tau p\right) \mathrm{e}^{\tau s_{ \pm}}}{2}, \\
k_{p}=-\frac{\left(\left(8 \tau+\tau^{2} s_{ \pm}\right) p-18-12 \tau s_{ \pm}\right) \mathrm{e}^{\tau s_{ \pm}}}{\tau}, \\
k_{i}=\frac{\left(\left(\tau s_{ \pm}+3\right) \tau^{2} p^{2}+\left(-12 \tau s_{ \pm}-60\right) \tau p+108+84 \tau s_{ \pm}\right) \mathrm{e}^{\tau s_{ \pm}}}{2 \tau^{2}} .
\end{array}\right.
$$

iii) If (8) is satisfied and $\left|k_{d}\right|<1$ then $s=s_{+}$is the rightmost root of quasipolynomial function (6).

Remark. - It is worth-noting that the degree of the quasipolynomial function (6) is equal to the generic Polya-Szegö bound denoted $P S_{B}=5$. Such a bound cannot be reached due to the sparsity of the polynomial $Q_{0}$.

- From a robustness point of view, the solution $s_{-}$is not of interest since in such a case the open loop system

$$
L_{0}(s)=P_{0}(s) K_{P I D}(s)
$$

do not satisfy the requirement for rolling off at high frequencies since $\left|L_{0}(\infty)\right|>1$, see for instance [9].

- If (8) is satisfied, then the quasipolynomial function (6) can be written as a Fredholm equation of the first type,

$$
\Delta(s)=\int_{0}^{1} \mathcal{K}(t, s) \phi(t) d t,
$$

where its smooth kernel function is given by:

$$
\mathcal{K}(t, s)=\mathrm{e}^{-t \tau\left(s-s_{+}\right)},
$$

and its solution to the inhomogeneous equation writes:

$$
\phi(t)=\frac{s_{+}\left(s_{+}-p\right)}{2} t^{2}+\frac{\left(2 s_{+}-p\right) t}{\tau}+\frac{1}{\tau^{2}} .
$$


In a $(p, \tau)$ parametrization such a solution is:

$$
\phi(t)=\frac{\left(12-3 \sqrt{\tau^{2} p^{2}+12}\right) t^{2}+\left(2 \sqrt{\tau^{2} p^{2}+12}-12\right)}{2 \tau^{2}}
$$

Other Fredholm equations of the first type appeared in [13], [20], [22] in investigating the dominant root of quasipolynomial functions corresponding to retarded delay systems. The specific polynomial structure of the solution to the inhomogeneous equation and the exponential structure of the kernel seem to be related to such a dominancy property. Such a question will be explored in a future work.

- In terms of the delay margin, the dominant root writes:

$$
s_{+}=\frac{-\left(\tau+3 \tau_{P I D}\right)+\sqrt{\tau^{2}+3 \tau_{P I D}{ }^{2}}}{\tau_{P I D} \tau} .
$$

Proof: [Sketch of the proof] The degree of the quasipolynomial function defined in (6) is equal to 5. So, using the Polya and Szegö result from [14] one asserts that 5 is the generic bound of the multiplicity of any root of (6). First, the vanishing of the quasipolynomial $\Delta$ given in (6) yields the elimination of the exponential term as a rational function in $s$ :

$$
\mathrm{e}^{-\tau s}=\frac{-s^{2}+p s}{k_{d} s^{2}+k_{p} s+k_{i}}
$$

Next, to investigate potential roots with algebraic multiplicity 4 , one substitutes of the obtained equality (15) in the ideal $\mathcal{I}_{4}$ generated by the first three derivatives of $\Delta$ that is $\mathcal{I}_{4}=<\partial_{s} \Delta, \partial_{s}^{2} \Delta, \partial_{s}^{3} \Delta>$. This allows to investigate the following variety of three algebraic equations in 6 unknowns $k_{i}, k_{p}, k_{d}, \tau, p, s:$

$$
\begin{aligned}
0= & \tau k_{d} s^{4}+\left(k_{p}-k_{d} p\right) \tau s^{3}+\left(\left(k_{i}-k_{p} p\right) \tau+k_{d} p+k_{p}\right) s^{2} \\
& +\left(2 k_{i}-\tau p k_{i}\right) s-p k_{i}, \\
0= & -\tau^{2} k_{d} s^{4}+\left(\left(-k_{p}+k_{d} p\right) \tau^{2}+4 \tau k_{d}\right) s^{3} \\
& +\left(\left(k_{p} p-k_{i}\right) \tau^{2}+\left(-4 k_{d} p+2 k_{p}\right) \tau\right) s^{2} \\
& +\left(\tau^{2} p k_{i}+2 k_{p}+2 k_{d} p-2 \tau k_{p} p\right) s+2 k_{i}, \\
0= & \tau^{3} k_{d} s^{4}+\left(\left(k_{p}-k_{d} p\right) \tau^{3}-6 \tau^{2} k_{d}\right) s^{3} \\
& +\left(\left(k_{i}-k_{p} p\right) \tau^{3}+\left(6 k_{d} p-3 k_{p}\right) \tau^{2}+6 \tau k_{d}\right) s^{2} \\
& +\left(-p k_{i} \tau^{3}+3 \tau^{2} k_{p} p-6 \tau k_{d} p\right) s
\end{aligned}
$$

Using standard elimination techniques, one obtains the following set of admissible solutions:

1) $k_{i}=k_{p}=k_{d}=0$.

2) $k_{i}=s=0$.

3) $k_{p}=-2 \frac{\left(\left(\tau s_{ \pm}+8\right) \tau p-12 \tau s_{ \pm}-18\right) k_{d}}{\tau\left(\tau\left(2 s_{ \pm}-p\right)+4\right)}$, $k_{i}=\frac{\left(\left(\tau s_{ \pm}+3\right) \tau^{2} p^{2}+\left(-12 \tau s_{ \pm}-60\right) \tau p+84 \tau s_{ \pm}+108\right) k_{d}}{\left(2 \tau s_{ \pm}+4-\tau p\right) \tau^{2}}$ where the explicit expression of $s_{ \pm}$is given by (7).

Observe that the first solution corresponds to the openloop system while the second solution is inconsistent with respect to the transcendental-term elimination (15). So that, these two solutions are discarded. Furthermore, substituting conditions of the third solution in (15) yields the explicit values of the gain $k_{d}$ allowing to tune the parameters as provided in (8).

$t+N 2$ te that, when forcing multiplicity 5 complex gains and delay are obtained. Since we deal only with quasipolynomials with real coefficients, such a multiplicity is discarded.

Next to show that $s_{+}$is the dominant root of the quasipolynomial $\Delta$ (with coefficients satisfying (8)), one has to apply the principle of the argument. However, it is necessary first to prove that only a finite number of roots of $\Delta$ may occur in the right-half complex plane $\mathbb{C}_{s_{+}}^{+}=\left\{s \in \mathbb{C}\right.$, s.t. $\left.\Re(s)>s_{+}\right\}$. According to Proposition 1, one has to check that $\tau s_{+}>$ $\log (|\alpha|)$, see also [28, Prop. 2.1]. In our case, $\alpha$ is nothing but $k_{d}$. So that,

$$
\begin{aligned}
\log \left(k_{d}\right) & =\log \left(\left(-1+1 / 2 \sqrt{\tau^{2} p^{2}+12}\right) \mathrm{e}^{-1 / 2 \tau p-3+1 / 2 \sqrt{\tau^{2} p^{2}+12}}\right) \\
& =\tau s_{+}-\log (2)+\log \left(-2+\sqrt{\tau^{2} p^{2}+12}\right)
\end{aligned}
$$

Since, $\tau<\tau_{P I D}=\frac{2}{p}$, then $\sqrt{\tau^{2} p^{2}+12}<4$. So that, $\log \left(k_{d}\right)<\tau s_{+}$which proves that only a finite number of roots of $\Delta$ may occur in the right-half complex plane $\mathbb{C}_{s_{+}}^{+}=$ $\left\{s \in \mathbb{C}\right.$, s.t. $\left.\Re(s)>s_{+}\right\}$.

Equivalently, by using the following scaling $s \rightarrow z+s_{+}$ and the new parametrization $\delta=\sqrt{\tau^{2} p^{2}+12}$, one has to show the dominancy of zero spectral value for the following quasipolynomial function:

$$
\begin{aligned}
\tilde{\Delta}(z)= & z^{2}+\frac{\delta-6}{\tau} z+\frac{12-3 \delta}{\tau^{2}} \\
& +\left(\frac{\delta-2}{2} z^{2}+\frac{2 \delta-6}{\tau} z+\frac{3 \delta-12}{\tau^{2}}\right) \mathrm{e}^{-z \tau}
\end{aligned}
$$

Obviously, $z=0$ is a root of (17) with multiplicity 4 . To apply the principle argument on the standard Bromwich contour, which allows to counting the roots of the quasipolynomial (17) on the right half-plane, one needs first to introduce a deflation eliminating the roots on the imaginary axis. To do so, let us first investigate nonzero imaginary roots for (17). Assume that there exists $\omega>0$ such that $z=i \omega$ is a root of (17). Let define $R(\omega)=\Re(\tilde{\Delta}(i \omega))$ and $S(\omega)=\Im(\tilde{\Delta}(i \omega))$, which gives :

$$
\left\{\begin{aligned}
R(\omega)= & \left(\frac{2-\delta}{2} \omega^{2}+\frac{3 \delta-12}{\tau^{2}}\right) \cos (\omega \tau) \\
& +\frac{(2 \delta-6) \omega \sin (\omega \tau)}{\tau}-\omega^{2}+\frac{12-3 \delta}{\tau^{2}} \\
S(\omega)= & \left(\frac{\delta-2}{2} \omega^{2}+\frac{12-3 \delta}{\tau^{2}}\right) \sin (\omega \tau) \\
& +\frac{\omega(2 \delta-6) \cos (\omega \tau)}{\tau}+\frac{\delta-6}{\tau} \omega
\end{aligned}\right.
$$

This means that for any $z=i \omega$ a root of (17) one has:

$$
\left\{\begin{array}{l}
R(\omega)=0 \\
S(\omega)=0
\end{array}\right.
$$

Some algebraic manipulations allows to eliminate the trigonometric functions. Next, using the standard trigonometric identity $\cos ^{2}(\omega \tau)+\sin ^{2}(\omega \tau)=1$ which is equivalent 


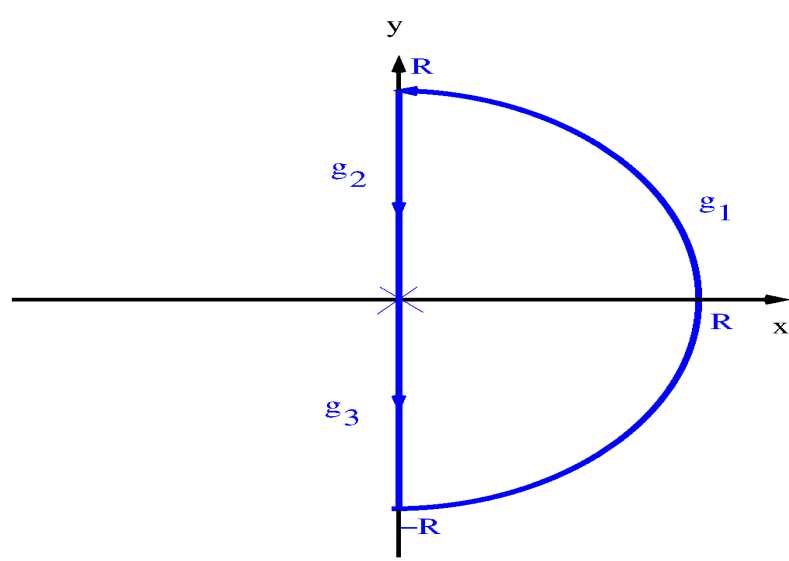

Fig. 2. The used contour for applying the argument principle to investigate the dominancy of the multiple root.

to

$0=\Omega^{2} \delta(\delta-4) \times$

$\left(\left(\delta^{2}-4 \delta+4\right) \Omega^{2}+\left(4 \delta^{2}-24 \delta+48\right) \Omega+36 \delta^{2}+576-28\right.$

where $\Omega=\tau^{2} \omega^{2}$. Since $\tau<\tau_{P I D}=2 / p$, then , one shows that no positive solution exists for both cases $\left(\star_{ \pm}\right)$.

Now, one is able to apply the principle of the argument to investigate the dominancy of $s_{+}$as a root of $\Delta$, given by (6) such that its coefficients satisfy (8), which is equivalent to investigate the dominancy of zero as a root of $\tilde{\Delta}$ given by (17). Clearly, apart from zero, $\tilde{\Delta}$ and $\hat{\Delta}(s)=\frac{\tilde{\Delta}(s)}{s^{4}}$ have the same roots. But it seems to be more computationally convenient to investigate roots of the deflated analytic function $\hat{\Delta}$ rather than $\tilde{\Delta}$. Let define the integration contour $\gamma=\cup_{k=1}^{2} C_{k}$ which is taken as a counterclockwise closed curve, then an integral over $\gamma$ is defined as the sum of the integrals over the directed smooth curves that make $\gamma$ up, as depicted in Figures 2.

Elementary calculations give parametrization of $\gamma$ :

$$
\left\{\begin{array}{l}
z_{1}(\theta)=R \mathrm{e}^{i \theta}, \quad \text { for } \quad-\frac{P i}{2} \leq \theta \leq \frac{P i}{2}, \\
z_{2}(t)=i t, \quad \text { for } \quad R \leq t \leq-R
\end{array}\right.
$$

where $z_{k}(t)$ designates the parametrization of $z$ along $C_{k}$ for $k \in\{1,2\}$. Since $\hat{\Delta}$ is analytic and dont vanish on the imaginary axis (including zero) then the argument principle asserts:

$$
\frac{1}{2 i \pi} \oint_{\gamma} \frac{\partial_{s} \hat{\Delta}(s)}{\hat{\Delta}(s)} d s=\mathcal{Z},
$$

where $\mathcal{Z}$ designates the number of the quasipolynomial roots enclosed by $\gamma$. Furthermore, the left-hand side of (21) gives:

$$
\begin{aligned}
\oint_{\gamma} \frac{\partial_{s} \hat{\Delta}(s)}{\hat{\Delta}(s)} d s=\lim _{R \rightarrow \infty} \sum_{k=1}^{2} I_{k}(t) \quad \text { where } \\
I_{1}(\theta)=\int_{-\frac{\pi}{2}}^{\frac{\pi}{2}} \dot{z}_{1}(\theta) \frac{\partial_{s} \hat{\Delta}\left(z_{1}(\theta)\right)}{\hat{\Delta}\left(z_{1}(\theta)\right)} d \theta, \\
I_{2}(t)=-\int_{-R}^{R} \dot{z}_{2}(t) \frac{\partial_{s} \Delta\left(z_{2}(t)\right)}{\Delta\left(z_{2}(t)\right)} d t .
\end{aligned}
$$

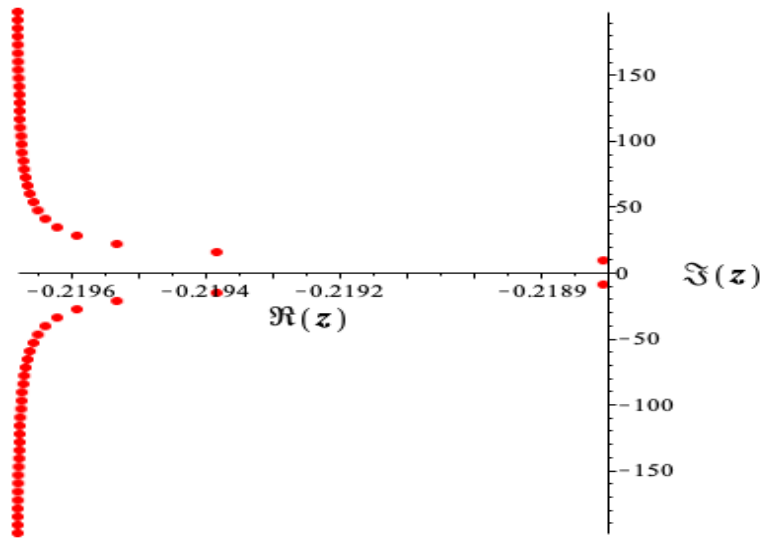

Fig. 3. The distribution of the spectrum corresponding to deflated characteristic function $\hat{\Delta}$ (6) for $p=\tau=1$ and the controller gains given in (23).

Since we are interested in the limit when $R$ goes to infinity, gne uses the uniform convergence theorem and then explores the dominant terms in $R$ respectively the numerator and the denominator of the integrants of $I_{1}$ and $I_{2}$. The last tedious step, consists in evaluating the limit of the resulting integrals $I_{1}$ and $I_{2}$ which gives $\mathcal{Z}=0$.

\section{ILLUSTRATIVE EXAMPLE}

Consider the plant depicted in Figure 1 where $P(s)$ is a delayed first order instable plant and $K(s)$ is a PID controller. So that the performance properties of the closedloop plant are characterized via the transfert function (5) and its stability is characterized by (6). Assume the $K(s)$ admits an instable pole at $p=1$ and a delay $\tau=1$. One tunes the controller gains as prescribed in (8) and such that $s_{+}$is a root of (6), which gives:

$$
\begin{aligned}
& k_{d}=1 / 2(-2+\sqrt{13}) \mathrm{e}^{-5 / 2+1 / 2 \sqrt{13}} \\
& k_{i}=1 / 2\left(-\frac{263}{2}+\frac{73}{2} \sqrt{13}\right) \mathrm{e}^{-5 / 2+1 / 2 \sqrt{13}} \\
& k_{p}=-\left(\frac{35}{2}-11 / 2 \sqrt{13}\right) \mathrm{e}^{-5 / 2+1 / 2 \sqrt{13}}
\end{aligned}
$$

This guarantees that $s_{+}=-5 / 2+1 / 2 \sqrt{13}$ is a root of the quasipolynomial function (6) with multiplicity 4 . Obviously, $s_{+}$is negative and its dominancy is asserted by Theorem III.1. Figure 3 exhibits the roots $z_{k}$ corresponding to $\hat{\Delta}$ which are commun roots with $\tilde{\Delta}$ apart from zero. So that, the roots of $\Delta$ are obtained by the scaling $s_{k}=z_{k}+s_{+}$apart from the quadruple root at $s_{+}$. Furthermore, since $\hat{\Delta}$ is analytic inside and on a closed contour $\gamma$, and is not zero on $\gamma$, then the number of its within $\gamma$ is equal to the number of times the image curve of $\gamma$ under the mapping $\omega=\hat{\Delta}(z)$ encircles the origin in the $\omega$-plane. The point $\omega=\hat{\Delta}(z)$ moves around as depicted in FigVar, where $\theta$ increases from $-\pi / 2$ to $\pi / 2$, and $y$ increases from $-R$ to $R$. Since the curve dont encircles the origin, the argument of $\hat{\Delta}$ dont increase, see for instance [1]. 


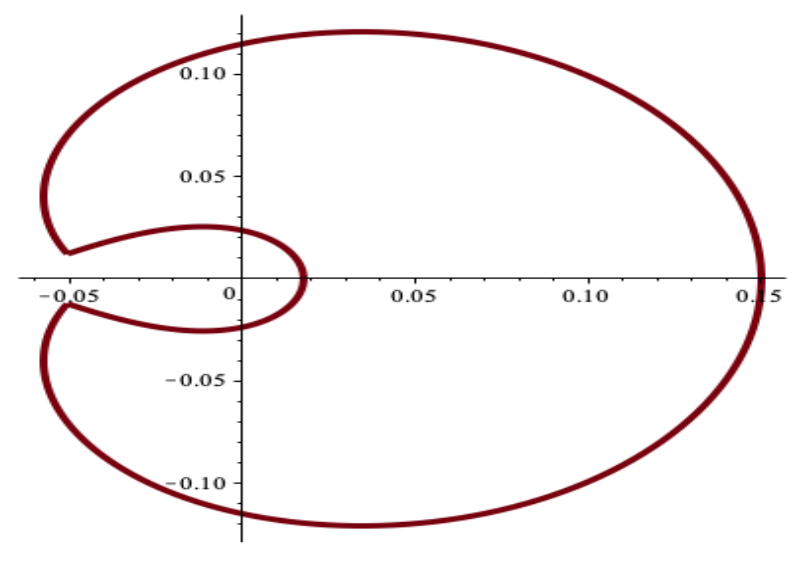

Fig. 4. The argument variation: the behavior the point $\omega$ defined by the mapping $\omega=\hat{\Delta}(z)$.

\section{CONCLUSION}

In this paper we investigated the effect of multiple spectral values on the stability of time-delay systems. An extension of the MID property to linear functional differential equations of neutral type is provided. We showed the potential applicability of such a property in a control design problem. The proposed approach yields a systematic tuning for PID controllers satisfying the requirement for rolling off at high frequencies.

\section{ACKNOWLEDGMENTS}

IB \& SIN are partially financially supported by a public grant overseen by the French National Research Agency (ANR) as part of the "Investissement d'Avenir" program, through the "iCODE Institute project" funded by the IDEX Paris-Saclay and a grant from Hubert Curien (PHC) BALATON, project number $40502 \mathrm{NM}$.

\section{REFERENCES}

[1] R. Bellman and K. Cooke, Differential-difference equations. New York: Academic Press, 1963.

[2] K. L. Cooke and P. van den Driessche, "On zeroes of some transcendental equations," Funkcial. Ekvac., vol. 29, no. 1, pp. 77-90, 1986.

[3] K. Walton and J. E. Marshall, "Direct method for tds stability analysis," IEE Proceedings D - Control Theory and Applications, vol. 134, no. 2, pp. 101-107, 1987.

[4] G. Stépán, Retarded Dynamical Systems: Stability and Characteristic Functions, ser. Pitman research notes in mathematics series. Longman Scientific and Technical, 1989.

[5] J. K. Hale and S. M. V. Lunel, Introduction to functional differential equations, ser. Applied Mathematics Sciences. Springer Verlag, New York, 1993, vol. 99.

[6] W. Michiels and S.-I. Niculescu, Stability and stabilization of timedelay systems, ser. Advances in Design and Control. SIAM, 2007, vol. 12.

[7] N. Olgac and R. Sipahi, "An exact method for the stability analysis of time delayed linear time-invariant (lti) systems." IEEE Transactions on Automatic Control, vol. 47, no. 5, pp. 793-797, 2002.

[8] R. Sipahi, S. i. Niculescu, C. T. Abdallah, W. Michiels, and K. Gu, "Stability and stabilization of systems with time delay," IEEE Control Systems, vol. 31, no. 1, pp. 38-65, Feb 2011.

[9] D. Ma and J. Chen, "Delay margin of low-order systems achievable by pid controllers," IEEE Transactions on Automatic Control, pp. 1-1, 2018.
[10] I. Boussaada and S.-I. Niculescu, "Tracking the algebraic multiplicity of crossing imaginary roots for generic quasipolynomials: A Vandermonde-based approach," IEEE Transactions on Automatic Control, vol. 61, pp. 1601-1606, 2016.

[11] _ _ "Characterizing the codimension of zero singularities for timedelay systems," Acta Applicandae Mathematicae, vol. 145, no. 1, pp. 47-88, 2016.

[12] _ - "Computing the codimension of the singularity at the origin for delay systems: The missing link with Birkhoff incidence matrices," 21 st International Symposium on Mathematical Theory of Networks and Systems, pp. $1-8,2014$.

[13] I. Boussaada, H. Unal, and S.-I. Niculescu, "Multiplicity and stable varieties of time-delay systems: A missing link," in Proceeding of the 22nd International Symposium on Mathematical Theory of Networks and Systems, 2016, pp. 1-6.

[14] G. Pólya and G. Szegô, Problems and Theorems in Analysis, Vol. I: Series, Integral Calculus, Theory of Functions. New York, Heidelberg, and Berlin: Springer-Verlag, 1972.

[15] J. Vanbiervliet, K. Verheyden, W. Michiels, and S. Vandewalle, "A nonsmooth optimisation approach for the stabilisation of time-delay systems," ESAIM: COCV, vol. 14, no. 3, pp. 478-493, 2008.

[16] A. Ramirez, S. Mondie, R. Garrido, and R. Sipahi, "Design of proportional-integral-retarded (pir) controllers for second-order lti systems," IEEE Transactions on Automatic Control, no. 99, pp. 1-6, 2015.

[17] T. Vyhlidal and P. Zitek, "Mapping based algorithm for large-scale computation of quasi-polynomial zeros," IEEE Transactions on Automatic Control, vol. 54, no. 1, pp. 171-177, 2009.

[18] T. Mori, N. Fukuma, and M. Kuwahara, "On an estimate of the decay rate for stable linear delay systems," International Journal of Control, vol. 36, no. 1, pp. 95-97, 1982.

[19] N. D. Hayes, "Roots of the transcendental equation associated with a certain difference-differential equation," Journal of the London Mathematical Society, vol. s1-25, no. 3, pp. 226-232, 1950.

[20] I. Boussaada, S. Tliba, S.-I. Niculescu, H. U. Unal, and T. Vyhlidal, "Further remarks on the effect of multiple spectral values on the dynamics of time-delay systems. application to the control of a mechanical system," Linear Algebra and its Applications, 2017.

[21] I. Boussaada, S.-I. Niculescu, S. Tliba, and T. Vyhlidal, "On the coalescence of spectral values and its effect on the stability of timedelay systems: Application to active vibration control," Procedia IUTAM, vol. 22, no. Supplement C, pp. 75 - 82, 2017.

[22] I. Boussaada and S.-I. Niculescu, "On the dominancy of multiple spectral values for time-delay systems with applications," in To appear in: Proceeding of The 14th IFAC Workshop on Time Delay Systems, 2018, pp. 1-6.

[23] W. Michiels, K. Engelborghs, P. Vansevenant, and D. Roose, "Continuous pole placement for delay equations," Automatica, vol. 38, no. 5, pp. 747 - 761, 2002.

[24] P. Zitek, J. Fiser, and T. Vyhlidal, "Dimensional analysis approach to dominant three-pole placement in delayed pid control loops," Journal of Process Control, vol. 23, no. 8, pp. 1063 - 1074, 2013.

[25] S.-I. Niculescu and W. Michiels, "Stabilizing a chain of integrators using multiple delays," IEEE Trans. on Aut. Cont., vol. 49, no. 5, pp. 802-807, 2004.

[26] I. Boussaada, D. Irofti, and S.-I. Niculescu, "Computing the codimension of the singularity at the origin for time-delay systems in the regular case: A Vandermonde-based approach," 13th European Control Conference, pp. 1-6, 2014.

[27] A. M. Krall, "On the real parts of zeros of exponential polynomials," Bull. Amer. Math. Soc., vol. 70, pp. 291-292, 1964.

[28] J. R. Partington and C. Bonnet, " $h_{\infty}$ and bibo stabilization of delay systems of neutral type," Systems \& Control Letters, vol. 52, no. 3, pp. $283-288,2004$.

[29] G. J. Silva, A. Datta, and S. P. Bhattacharyya, "New results on the synthesis of pid controllers," IEEE Transactions on Automatic Control, vol. 47, no. 2, pp. 241-252, 2002. 